\title{
Método de pontos interiores para estimar os parâmetros de um modelo probabilístico usando o corpus Thyco Brahe
}

\author{
Esther S. Mamián Lopez ${ }^{1}$, Aurelio Ribeiro Leite Oliveira ${ }^{1}$ \\ ${ }^{1}$ Inst. de Matemática, Estatística e Computação Científica - Universidade Estadual de \\ Campinas (UNICAMP) \\ Rua Sérgio Buarque de Holanda, 651- 13083-859- Campinas - SP - Brazil \\ esmamian@gmail.com, aurelio@ime.unicamp.br
}

\begin{abstract}
Statistical methods research for natural language processing and other important applications, have been presenting fast growth in the recent years. In this work, we propose a primal-dual interior point method for trainning stochastic context free grammar. For that purpose, we use a Portuguese based corpus Tycho Brahe [IEL-UNICAMP and IME-USP ].
\end{abstract}

Resumo. Nos anos recentes têm acontecido um importante crescimento no interesse sobre os métodos estatísticos para processamento de linguagens $e$ as diferentes aplicações que se derivam. Propomos um método de pontos interiores barreira logarítmica primal-dual para abordar o problema de atribuir valores ótimos de probabilidade às regras de uma gramática probabilística livre de contexto (GPLC) baseados no corpus da linguagem portuguesa Tycho Brahe [IEL-UNICAMP and IME-USP ].

\section{Introdução}

Dentre os modelos estatísticos para modelar as linguagens naturais estão as gramáticas probabilísticas livres de contexto, que na sua forma mais simples podem ser decompostas numa parte estrutural e numa parte estocástica. Abordamos o problema de modelar uma linguagem natural através de uma GPLC treinando a mesma, ou seja, encontrar as probabilidades ótimas associadas às regras da gramática [Manning and Schutze. 2003]. Este processo é realizado em base a um corpus que contém sentenças da linguagem que desejamos modelar. Para o processo do treino precisamos de uma função critério da amostra ${ }^{1}$ e um marco para otimizá-la: usamos a função de máxima verossimilhança da amostra e o método de pontos interiores barreira logarítmica primal-dual, respectivamente.

Assim, o problema de treinamento de uma gramática é resumido como o problema de otimização descrito a seguir:

$$
\begin{array}{cll}
\underset{\text { sujeito a }}{\operatorname{maximizar}} & f(x) & \sum_{x_{i} \in \Psi_{A}} x_{i}=1, \quad \forall \Psi_{A}: A \in \Sigma \\
& 0 \leq x_{i} \leq 1, \quad i=1, \ldots,|P|
\end{array}
$$

onde $f(x)=\operatorname{Pr}\left(\Omega \mid G_{p}\right)$ é a função de verossimilhança que, depende da amostra $\Omega$ e da GPLC $G_{p}$. Esta função é um polinômio nas variáveis $x_{i}, i=1 \ldots|P|$, sendo que

\footnotetext{
${ }^{1}$ Usamos a palavra corpus e amostra indistintamente para nos referir ao conjunto de sentenças de uma linguagem natural que estamos desejando modelar.
} 
$x_{i}$ correspondem aos valores de probabilidade associados às regras de $G_{p}$. Denotamos por $\Psi_{A}$ como os valores de probabilidade associados às regras que possuem o mesmo antecedente. Denotamos por $\Sigma$ o conjunto finito de símbolos não terminais, e $P$ como o conjunto finito das regras da $G_{p}$.

\section{Metodologia}

Uma vez definido o problema (1), implementamos o método barreira logarítmica primal-dual baseados na formulação para problemas não lineares, descrita em [El-Bakry et al. 1996]. Este método foi implementado na linguagem C++, versão 4.8.4. O corpus usado é o Tycho Brahe, para o português do Brasil. Para analisar a viabilidade da proposta extraímos do corpus algumas GPLCs de diferentes tamanhos e as treinamos usando o método barreira logarítmica primal-dual. Seguem os detalhes das gramáticas extraídas para o treinamento e algumas discussões associadas às implementações.

\subsection{Tamanho dos problemas}

Trabalhamos com seis sub-problemas detalhados na Tabelas 1 e 2.

\begin{tabular}{|l|r|r|r|c|}
\hline & $\begin{array}{c}\text { Nro símbolos } \\
\text { não terminais } m\end{array}$ & $\begin{array}{c}\text { Nro símbolos } \\
\text { terminais } n\end{array}$ & $\begin{array}{c}\text { Nro de regras } \\
|P|\end{array}$ & Símbolo inicial \\
\hline Sub-Gramática 1 & 5 & 2.500 & 12.580 & $\mathrm{~S}$ \\
\hline Sub-Gramática 2 & 7 & 2.500 & 17.752 & $\mathrm{~S}$ \\
\hline Sub-Gramática 3 & 9 & 2.500 & 23.076 & $\mathrm{~S}$ \\
\hline
\end{tabular}

Tabela 1. Características da gramática para cada sub-problema do 1 até 3.

\begin{tabular}{|l|r|r|r|c|}
\hline & $\begin{array}{c}\text { Nro símbolos } \\
\text { não terminais } m\end{array}$ & $\begin{array}{c}\text { Nro símbolos } \\
\text { terminais } n\end{array}$ & $\begin{array}{c}\text { Nro de regras } \\
|P|\end{array}$ & Símbolo inicial \\
\hline Sub-Gramática 4 & 3 & 5.500 & 16.512 & $\mathrm{~S}$ \\
\hline Sub-Gramática 5 & 3 & 7.500 & 22.512 & $\mathrm{~S}$ \\
\hline Sub-Gramática 6 & 3 & 7.500 & 22.512 & $\mathrm{~S}$ \\
\hline
\end{tabular}

Tabela 2. Características da gramática para cada sub-problema do 4 até 6.

\subsection{Cálculo da função objetivo $f(x)$}

Note que, o cálculo da função objetivo $f(x)$ deve ser feita a cada iteração do método de pontos interiores barreira logarítmica primal-dual. E, dado que a função objetivo depende das probabilidades das sentenças do corpus, observe que não é eficiente calcular a probabilidade de uma sentença como a soma das probabilidades de todas suas possíveis árvores sintáticas. Para isso, implementamos um método baseado no método Cocke-YoungerKassami, desenvolvido em [López 2018] para calcular o valor da função objetivo sem comprometer as capacidades físicas das máquinas, e utilizamos a biblioteca Open Multiprocessing (OpenMP 2.5) para melhorar os tempos computacionais deste método.

\subsection{Cálculo do gradiente e da Hessiana de $f(x)$}

Os valores do gradiente e da Hessiana devem ser calculados a cada iteração. Usamos o método das diferenças finitas para calculá-lo, pois a expressão das derivadas é muito cara. 


\subsection{Resolução do sistema linear}

Além do custo computacional para calcular o valor da função objetivo e suas derivadas a cada iteração, o trabalho computacional dos métodos de pontos interiores também é dominado pela resolução de um sistema linear [Gondzio 2012]. Portanto uma forma eficiente de solução desses sistemas lineares é indispensável para resolver problemas de grande porte.

A matriz de coeficientes do sistema [Gondzio 2012] é uma matriz indefinida, mal condicionada, esparsa, portanto usamos um método iterativo precondicionado: o método do gradiente bi-conjugado estabilizado para matrizes esparsas, com precondicionador baseado nas entradas da diagonal [Saad 2003].

\subsection{Reescalonamento do problema}

Uma grande dificuldade é quando, no método de pontos interiores barreira logarítmica primal-dual, os diferentes valores das variáveis com as quais estamos trabalhando atingem valores pequenos causando underflow, levando a problemas estabilidade e arredondamento [Trefethen and Bau III 1997, Ruggiero and da Rocha Lopes. 1997]. Dada a ordem do polinômio do problema, os principais valores que devemos analisar e acompanhar são a função de verossimilhança, o valor do gradiente e o valor da Hessiana. Portanto, nossa proposta é manter os valores dessas grandezas e os cálculos que os envolvem controlados, tanto para diminuir os erros de arredondamento, como para evitar problemas de underflow. Assim, quando for preciso, multiplicamos a função objetivo por uma constante $C \gg 0$. Basicamente estamos re-escalando os valores numéricos para resolver estes inconvenientes.

\section{Resultados e discussões}

Nas tabelas a seguir apresentamos os resultados obtidos para as seis sub-gramáticas detalhadas nas Tabelas 1 e 2 . Apresentamos o tamanho da amostra usada, o número de iterações e o tempo que utilizou o método até convergir, assim como o valor da constante $C$. Para as primeiras três sub-gramáticas (ver Tabela 1) usamos uma amostra de tamanho 67, com comprimento das sentenças entre quatro e cinco. Para o segundo grupo de sub-gramáticas (ver Tabela 2) usamos amostras de diferentes tamanhos e sentenças de comprimento entre quatro e quinze.

\begin{tabular}{|l|c|c|r|c|}
\hline & $\|\Omega\|$ & $\begin{array}{c}\text { Iterações } \\
\text { até convergir }\end{array}$ & \multicolumn{1}{|c|}{ Tempo } & Vlr da constante \\
\hline Sub-Problema 1 & 67 & 9 & $34 \mathrm{~m} 45,830 \mathrm{~s}$ & $10^{20}$ \\
Sub-Problema 2 & 67 & 9 & $335 \mathrm{~m} 13,625 \mathrm{~s}$ & $10^{15}$ \\
Sub-Problema 3 & 67 & 9 & $4558 \mathrm{~m} 27,323 \mathrm{~s}$ & $10^{10}$ \\
\hline
\end{tabular}

Tabela 3. Resultados obtidos para o primeiro grupo de sub-problemas.

Da Tabela 3 podemos evidenciar que, uma vez obtida a convergência para um problema com $m$ símbolos não terminais, podemos aumentar este valor e utilizando a estratégia para controlar problemas de underflow, atingimos a convergência do método.

A Tabela 4 apresenta um aumento do tamanho da amostra e do comprimento das sentenças da mesma, havendo um maior tamanho dos problemas. O método atinge convergência e 


\begin{tabular}{|l|c|c|c|c|}
\hline & $\|\Omega\|$ & $\begin{array}{c}\text { Iterações } \\
\text { até convergir }\end{array}$ & Tempo & $\begin{array}{c}\text { Vlr da constante } \\
\text { C }\end{array}$ \\
\hline Sub-Problema 4 & 161 & 9 & $127 \mathrm{~m} 19,235 \mathrm{~s}$ & $10^{25}$ \\
Sub-Problema 5 & 388 & 9 & $407 \mathrm{~m} 19,538 \mathrm{~s}$ & $10^{25}$ \\
Sub-Problema 6 & 310 & 9 & $9519 \mathrm{~m} 2,029 \mathrm{~s}$ & $10^{15}$ \\
\hline
\end{tabular}

Tabela 4. Resultados obtidos para o segundo grupo de sub-problemas.

como no caso do primeiro grupo de testes, o acompanhamento garante que não se gerem problemas de underflow, isto basicamente é controlado com o parâmetro $C$.

\section{Conclusão}

O método de pontos interiores mostrou-se um método viável para estimar as gramáticas probabilísticas livres do contexto. Um dos detalhes relevantes é que o número de iterações até atingir convergência é mantido em nove iterações, isto quer dizer que o método é estável para diferentes tamanhos tanto da gramática como do corpus. Isto é importante porque uma das maiores desvantagens do Método Inside-Outside [Baker 1979] em aplicações práticas é o elevado número de iterações requeridas para atingir a convergência.

O método de pontos interiores implementado é robusto e bem comportado na presença de novos dados, sendo uma proposta que sugere continuar fazendo testes aumentando o tamanho da amostra, até conseguir modelar uma linguagem natural.

\section{Referências}

Baker, J. K. (1979). Trainable grammars for speech recognition. The Journal of the Acoustical Society of America, 65(S1):S132-S132.

El-Bakry, A., Tapia, R., Tsuchiya, T., and Zhang, Y. (1996). On the formulation and theory of the newton point-pnterior for nonlinear programming. Journal of Optimization Theory and Applications.

Gondzio, J. (2012). Interior point methods 25 years later. European Journal of Operational Research, 218(3):587-601.

IEL-UNICAMP and IME-USP. Corpus anotado do Português histórico Tycho Brahe. http://www.tycho.iel.unicamp.br/corpus/index.html, acessado em 2017.

López, E. S. M. (2018). Método de pontos interiores para estimar os parâmetros de uma gramática probabilística livre do contexto. Disertação doutorado, Universidade Estadual de Campinas. Instituto de matemática, Estatística e Computação Científica.

Manning, C. D. and Schutze., H. (2003). Foundations of statistical natural language processing. Cambridge, MA : MIT.

Ruggiero, M. A. G. and da Rocha Lopes., V. L. (1997). Cálculo numérico. Aspectos teóricos e computacionais. São Paulo, SP : Makron.

Saad, Y. (2003). Iterative Methos for Sparse Linear Systems. SIAM Publications, SIAM, Philadelphia, PA, USA.

Trefethen, L. N. and Bau III, D. (1997). Numerical linear algebra. Siam. 